\title{
ON CYCLIC SUBGROUPS AND THE CONJUGACY PROBLEM
}

\author{
R. DANIEL HURWITZ
}

\begin{abstract}
The conjugacy problem in three types of group constructions involving cyclic subgroups is discussed. First it is shown that if $G$ has the solvable conjugacy problem and if $h \in G$ and $k \in G$ satisfy (a) $h$ and $k$ are not power conjugate to themselves or each other, (b) the power conjugacy problem in $G$ with respect to $h$ or $\boldsymbol{k}$ is solvable, and (c) the double coset solvability problem in $\boldsymbol{G}$ is solvable with respect to $\langle h\rangle$ and $\langle k\rangle$, then the HNN extension $G^{*}=\left\langle G, t ; t^{-1} h t=k\right\rangle$ has the solvable conjugacy problem. This result is used to deduce a similar theorem for free products with amalgamation, a fact first stated by Lipschutz. Then it is shown that if $A$ and $B$ are groups with the solvable conjugacy problem and $h \in A$ and $k \in B$ taken with themselves satisfy the conditions above in $A$ and $B$, respectively, then $\langle A * B ;[h, k]=1\rangle$ has the solvable conjugacy problem.
\end{abstract}

1. Introduction. The conjugacy problem in free products with cyclic amalgamated subgroups and in HNN extensions with cyclic associated subgroups has received a good deal of attention from various authors. Lipschutz [7] solved the conjugacy problem for the free product of free groups with cyclic amalgamated subgroups. Lipschutz [8] also proved that free products with cyclic amalgamations have the solvable conjugacy problem assuming that the factors have the solvable conjugacy problem and that the generators of the amalgamated cyclic subgroups are of a certain type, which he called semicritical.

Anshel and Stebe [2] first solved the conjugacy problem for an HNN extension of a free group with isomorphic cyclic associated subgroups. Anshel [1] extended this result to certain HNN groups having many stable letters.

Comerford and Truffault [4] solved the conjugacy problem in free products with cyclic amalgamations and HNN extensions with isomorphic cyclic associated subgroups where the factors or base groups, respectively, are small cancellation groups (specifically, sixth-groups). Their main technical result shows that elements of sixth-groups are semicritical for the free product with the amalgamation case and what we will call mutually semicritical in the $\mathrm{HNN}$ case.

Throughout this paper we will use the symbol $\tilde{H}$ for the conjugacy relation in the group $H$. When it is clear which group is meant, we will write $\sim$.

Following Lipschutz [8], we make the following definitions: Let $G$ be a group, $h$ and $k$ elements of $G$. We will say that $h$ and $k$ are mutually semicritical if the following three conditions are satisfied:

(a) $h^{n} \sim h^{m}$ implies $n=m, k^{n} \sim k^{m}$ implies $n=m$, and $h \neq k$ implies $h^{n} \nsim k^{m}$ for all integers $m$ and $n$,

Received by the editors October 23, 1978.

AMS (MOS) subject classifications (1970). Primary 20F05; Secondary 20E05.

(c) 1980 American Mathematical Society 0002-9939/80/0000-0200/\$03.00 
(b) for all $w \in G$, we can decide whether or not $w$ is conjugate to a power of $h$, and the same for $k$,

(c) for any $u \in G, v \in G$, we can decide whether or not there exist integers $r$ and $s$ such that $x^{r} u y^{s}=v$ for all pairs $(x, y) \in\{h, k\} \times\{h, k\}$. We will say that $h$ is semicritical if $h$ is mutually semicritical with itself.

In this paper we will show:

TheOREM 1. Let $G$ have the solvable conjugacy problem and let $h$ and $k$ be mutually semicritical elements of $G$. Then the $H N N$ extension $G^{*}=\left\langle G, t ; t^{-1} h t=\right.$ $k>$ has the solvable conjugacy problem.

Then, using an argument from [5], we show how one may deduce Lipschutz's main result in [8] from Theorem 1. Finally, using the proof of Theorem 1 and the theory developed in [6], we show:

THEOREM 2. Let $A$ and $B$ be groups with the solvable conjugacy problem, and let $h$ and $k$ be semicritical elements of $A$ and $B$, respectively. Then the free product of $A$ and $B$ with commuting cyclic subgroups generated by $h$ and $k$ has the solvable conjugacy problem. We denote this group by $C=\langle A * B ;[h, k]=1\rangle$.

\section{Proof of Theorem 1.}

Proof. Suppose $u$ and $v$ are words of $G^{*}, u \nsim_{G} v$.

Case 1. Suppose $u$ and $v$ are $t$-free. Then by Collins' Lemma [3] there is a sequence $u=u_{0}, u_{1}, \ldots, u_{l}=v$ such that $u_{1}, \ldots, u_{l-1}$ are each in $\langle h\rangle$ or $\langle k\rangle$ and $u_{i+1}$ is obtained from $u_{i}$ by conjugating by an element of $G$ into the cyclic associated subgroups and then by $t^{ \pm 1}$ for $i=0, \ldots, l-1$.

If $u \nsim_{G} h^{\alpha}$ for some $\alpha$ or $u \nsim_{G} k^{\beta}$ for some $\beta$, or similarly for $v$, then $u \nsim_{G^{*}} v$. We may decide this by (b). So suppose we determine that $u \sim_{G} x^{\alpha}, v \sim_{G} y^{\beta}$ for some $x, y \in\{h, k\}$ and $\alpha, \beta \in \mathbf{Z}$. The $\alpha$ and $\beta$ are unique by (a). Conjugating by elements of $G$ cannot change $\alpha$ and $\beta$ because of (a), and conjugating by $t^{ \pm 1}$ also keeps the powers fixed. So $u \sim_{G^{*}} v$ if and only if $\alpha=\beta$ and $x=y$.

Case 2. If either $u$ or $v$ has a $t$-symbol, they must be $t$-circumparallel by Collins' Lemma. We choose cyclic conjugates in $G *\langle t\rangle$ of form $u^{*}=u_{1} t^{e_{1}} \cdots u_{n} t^{\ell_{n}}$ and $v^{*}=v_{1} t^{\varepsilon_{1}} \ldots v_{n} t^{\varepsilon_{n}}$ where $u_{i}, v_{i} \in G$ and $\varepsilon_{i}= \pm 1$ for $i=1, \ldots, n$. Of course $u^{*}$ and $v^{*}$ may effectively be determined from $u$ and $v$.

As shown by Anshel and Stebe [2], $u \sim_{G^{*}} v$ if and only if there is an integral solution $\alpha_{1}, \ldots, \alpha_{n}$ to the following system (*) of equations in $G$ :

$$
\begin{array}{ccc}
u_{1} y_{1}^{\alpha_{1}} & = & x_{n}^{\alpha_{n}} v_{1} \\
u_{2} y_{2}^{\alpha_{2}} & = & x_{1}^{\alpha} v_{2} \\
\vdots & & \vdots
\end{array} \text { where } x_{i}=\left\{\begin{array}{l}
h \\
k
\end{array}\right\} \text { and } y_{i}=\left\{\begin{array}{l}
k \\
h
\end{array}\right\} \text { if } \varepsilon_{i}=\left\{\begin{array}{c}
-1 \\
+1
\end{array}\right\}
$$

We will call an equation in (*) mixed if the $x_{i}$ and $y_{j}$ in that equation are different. 
Suppose a mixed equation of (*) has a solution, say $u_{i} h^{\alpha}=k^{\beta} v_{i}$. If also $u_{i} h^{\gamma}=k^{\delta} v_{i}$, then $u_{i}^{-1} k^{\delta-\beta} u_{i}=h^{\gamma-\alpha}$, so we must have $\beta=\delta$ and $\gamma=\alpha$ by (a). Thus, a solution of a mixed equation of (*) is unique. Moreover, a fixed solution to one equation in (*) allows us effectively to determine if $(*)$ has a solution, since we then successively have equations of form $x_{i}^{\alpha}=C$ where $C$ is a fixed word of $G$, and these equations may be uniquely determined by (b) and (a). Thus, if (*) has a mixed equation, we may determine if this equation has a solution using (c), and from this we may proceed to determine if (*) has a solution.

The remaining case occurs when all equations in (*) are not mixed. Suppose without loss of generality that the first equation is of form $u_{1} h^{\alpha_{1}}=h^{\alpha} v_{1}$. Then (*) must be of the following form:

$$
\begin{array}{ccc}
u_{1} h^{\alpha_{1}} & = & h^{\alpha} v_{1} \\
u_{2} k^{\alpha_{2}} & = & k^{\alpha} v_{2} \\
\vdots & & \vdots \\
u_{n-1} h^{\alpha_{n-1}} & = & h^{\alpha_{n-2}} v_{n-1} \\
u_{n} k^{\alpha_{n}} & = & k^{\alpha_{n-1}} v_{n} .
\end{array}
$$

Note that this may only occur if $n$ is even and the $t$-projection of $u$ and $v$ alternating; otherwise (*) has a mixed equation.

Rewriting, we get

$$
\begin{array}{ccc}
h^{-\alpha_{n}} u_{1} h^{\alpha_{1}} & = & v_{1} \\
k^{-\alpha_{1}} u_{2} k^{\alpha_{2}} & = & v_{2} \\
\vdots & & \vdots \\
h^{-\alpha_{n-2} u_{n-1} h^{\alpha_{n-1}}} & = & v_{n-1} \\
k^{-\alpha_{n-1}} u_{n} k^{\alpha_{n}} & = & v_{n} .
\end{array}
$$

By (c) we may determine whether or not there exist integers $p_{i}, q_{i}$ such that

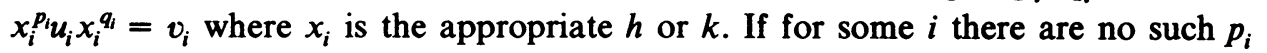
and $q_{i}$, then (**) has no solution. So we now suppose we have found $p_{1}, \ldots, p_{n}$,

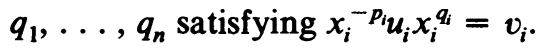

First of all we determine, by (c), if there are integers $\beta_{i}, \beta_{i}^{\prime}$ such that $x_{i}^{\beta_{i}} u_{i} x_{i}^{\beta_{i}^{\prime}}=$ $u_{i}$. If so, we must have $\beta_{i}^{\prime}=-\beta_{i}$ by (a). So if there is such a $\beta_{i}$, let $x_{i}^{\gamma_{i}}$ be a generator of the subgroup of $\langle h\rangle$ or $\langle k\rangle$ commuting with $u_{i}$. Otherwise, let $\gamma_{i}=0$. We may effectively determine $\gamma_{i}$ for $i=1, \ldots, n$.

If $x_{i}^{-r_{i}} u_{i} x_{i}^{s_{i}}=v_{i}$ then $u_{i}^{-1} x_{i}^{r_{i}-p_{i}} u_{i}=x_{i}^{s_{i}-q_{i}}$, so $r_{i}-p_{i}=s_{i}-q_{i}$ by (a). This means $x_{i}^{r_{i}-p_{i}} \in\left\langle x_{i}^{\gamma_{i}}\right\rangle$. Thus we have $x_{i}^{-r_{i}} u_{i} x_{i}^{s_{i}}=v_{i}$ if and only if $r_{i}=p_{i}+m_{i} \gamma_{i}$ and $s_{i}=q_{i}+m_{i} \gamma_{i}$ for some integer $m_{i}$.

Note that if $\gamma_{i}=0$ for some $i$, then the $i$ th equation of (**) has a unique solution $\left(p_{i}, q_{i}\right)$, and we may proceed to determine whether or not $(*)$ has a solution.

So suppose $\gamma_{i} \neq 0$ for $i=1, \ldots, n$. Then (*) has a solution $\left(\alpha_{1}, \ldots, \alpha_{n}\right)$ if and only if the following system $(* * *)$ of equations in $\mathbf{Z}$ has a solution $\left(m_{1}, \ldots, m_{n}\right)$ in $\mathbf{Z}$ : 


$$
\begin{array}{ccc}
q_{1}+m_{1} \gamma_{1} & = & p_{2}+m_{2} \gamma_{2} \\
q_{2}+m_{2} \gamma_{2} & = & p_{3}+m_{3} \gamma_{3} \\
\vdots & & \vdots \\
\vdots & & \vdots \\
q_{n-1}+m_{n-1} \gamma_{n-1} & = & p_{n}+m_{n} \gamma_{n} \\
q_{n}+m_{n} \gamma_{n} & = & p_{1}+m_{1} \gamma_{1} .
\end{array}
$$

Solving these equations successively for $m_{i}$, we find that $(* * *)$ has a solution if and only if $\sum_{j=1}^{n} p_{j}=\sum_{j=1}^{n} q_{j}$ and the following quantity is integral for $i=$ $2, \ldots, n$ :

$$
m_{i}=\left(m_{1} \gamma_{1}-\sum_{j=2}^{i} p_{j}-\sum_{j=1}^{i-1} q_{j}\right) / \gamma_{i}
$$

But $m_{1} \gamma_{1} \equiv \sum_{j=2}^{i} p_{j}-\sum_{j=1}^{i-1} q_{j}\left(\bmod \gamma_{i}\right)$ for $i=2, \ldots, n$ if $m_{1} \gamma_{1} \equiv \sum_{j=2}^{i} p_{j}-$ $\sum_{j=1}^{i-1} q_{j}\left(\bmod \Pi_{i=1}^{n} \gamma_{1}\right)$ for $i=2, \ldots, n$, so we need only check the elements in a complete residue system modulo $\Pi_{i=1}^{n} \gamma_{i}$ to determine if the system of congruences has a solution. This completes the proof.

The following lemma establishes that the standard embedding of a free product with amalgamation into the corresponding HNN extension is conjugacy preserving.

LEMMA. If $A_{i}, i \in I$, are groups with subgroups $B_{i}, i \in I$, and $\psi_{i}: B_{i} \rightarrow B_{i_{0}}$ are isomorphisms for $i \in I$, some fixed $i_{0} \in I$, let $J=I-\left\{i_{0}\right\}$. Let $H$ be the free product of the $A_{i}, i \in I$, amalgamating the subgroup $B_{i}$ via the isomorphisms $\psi_{i}$. Let $G^{*}$ be the analogously formed $H N N$ extension of the free product $P$ of the $A_{i}, i \in I$, with stable letters $t_{i}, i \in J$, that is,

$$
\begin{aligned}
H & =\left\langle P ; b_{i}=\psi_{i}\left(b_{i}\right) \text { for all } b_{i} \in B_{i},(i \in J)\right\rangle, \\
G^{*} & =\left\langle P, t_{i},(i \in J) ; t_{i}^{-1} b_{i} t_{i}=\psi_{i}\left(b_{i}\right) \text { for all } b_{i} \in B_{i},(i \in J)\right\rangle .
\end{aligned}
$$

Let $\varphi: H \rightarrow G^{*}$ be the embedding defined by $\varphi(h)=h$ if $h \in A_{i_{0}}, \varphi(h)=t_{i}^{-1} h t_{i}$ if $h \in A_{i}$ for $i \in J$. Then $\varphi$ has the property that, for all $h_{1}, h_{2} \in H, h_{1} \sim_{H} h_{2}$ if and only if $\varphi\left(h_{1}\right) \sim_{G^{*}} \varphi\left(h_{2}\right)$.

Proof. The 'only if' part is trivial. We prove the 'if' part. Suppose $h_{1}$ and $h_{2}$ are reduced words of $H$ and $\varphi\left(h_{1}\right) \sim_{G^{*}} \varphi\left(h_{2}\right)$. By Britton's Lemma, since $h_{1}$ and $h_{2}$ are reduced in $H$ (in the free product with amalgamation sense), $\varphi\left(h_{1}\right)$ and $\varphi\left(h_{2}\right)$ are reduced in $G^{*}$ (in the HNN sense).

Claim. We may assume $h_{1}$ and $h_{2}$ are cyclically reduced in $P$.

Proof OF CLAIM. If $\varphi\left(\tilde{h}_{1}\right) \sim_{G^{*}} \varphi\left(\tilde{h}_{2}\right)$ implies $\tilde{h}_{1} \sim_{H} \tilde{h}_{2}$ for cyclically reduced $\tilde{h}_{1}$ and $\tilde{h}_{2}$, suppose $h_{1}=c_{1}^{-1} \tilde{h}_{1} c_{1}$ in $P$ and $h_{2}=c_{2}^{-1} \tilde{h}_{2} c_{2}$ in $P$ where $\tilde{h}_{1}$ and $\tilde{h}_{2}$ are cyclically reduced in $P$. Then $\varphi\left(h_{1}\right) \sim_{G^{*}} \varphi\left(h_{2}\right)$ implies

$$
\varphi\left(c_{1}\right)^{-1} \varphi\left(\tilde{h}_{1}\right) \varphi\left(c_{1}\right) \sim_{G^{*}} \varphi\left(c_{2}\right)^{-1} \varphi\left(\tilde{h}_{2}\right) \varphi\left(c_{2}\right),
$$

so $\varphi\left(\tilde{h_{1}}\right) \sim_{G^{*}} \varphi\left(\tilde{h_{2}}\right)$. This implies $\tilde{h_{1}} \sim_{H} \tilde{h}_{2}$, and thus $h_{1} \sim_{H} h_{2}$. The claim is proved.

Write $h_{1}=p_{1} \cdots p_{n}$ where $p_{k} \in A_{j_{k}}$ for some $j_{k} \in I, k=1, \ldots, n$, and $p_{k} \notin B_{j_{k}}$ for $k=2, \ldots, n$. Similarly write $h_{2}=q_{1} \cdots q_{m}$. Then $\varphi\left(h_{1}\right)=$ $t_{j_{1}}^{-1} p_{1} t_{j_{1}} \cdots t_{j_{n}}^{-1} p_{n} t_{j_{n}}$ and $\varphi\left(h_{2}\right)=t_{l_{1}}^{-1} q_{1} t_{l_{1}} \cdots t_{l_{m}}^{-1} q_{m} t_{l_{m}}$ with $t_{i_{0}}=1$. 
Case 1. Suppose either $\varphi\left(h_{1}\right)$ or $\varphi\left(h_{2}\right)$ involve a $t$-symbol. By Collins' Lemma, $\varphi\left(h_{1}\right)$ and $\varphi\left(h_{2}\right)$ are $t$-circumparallel and $\varphi\left(h_{1}\right)^{*}=e^{-1} \varphi\left(h_{2}\right)^{*} e$ where $\varphi\left(h_{1}\right)^{*}$ and $\varphi\left(h_{2}\right)^{*}$ are cyclic conjugates of $\varphi\left(h_{1}\right)$ and $\varphi\left(h_{2}\right)$, respectively, both ending in $t_{i_{d}}^{e}$ for some $i_{d} \in J, \varepsilon= \pm 1$. Also, $e \in A_{i_{d}}$ if $\varepsilon=-1$ and $e \in A_{i_{0}}$ if $\varepsilon=1$. If $\varepsilon=1$, we have in $G^{*}$

$$
e^{-1} t_{j_{k}}^{-1} p_{k} t_{j_{k}} \cdots t_{j_{k-1}}^{-1} p_{k-1} t_{j_{k-1}} e=t_{l_{r}}^{-1} q_{r} t_{l_{r}} \cdots t_{l_{r-1}}^{-1} q_{r-1} t_{l_{r-1}}
$$

This is exactly $\varphi\left(e^{-1} p_{k} \cdots p_{k-1} e\right)=\varphi\left(q_{r} \cdots q_{r-1}\right)$. Since $\varphi$ is an embedding, we conclude $e^{-1} p_{k} \cdots p_{k-1} e=q_{r} \cdots q_{r-1}$ in $H$. This gives $p_{k} \cdots p_{k-1}$ $\sim_{H} q_{r} \cdots q_{r-1}$, from which we have $h_{1} \sim_{H} h_{2}$.

If $\varepsilon=-1$, we have in $G^{*}$

$$
e^{-1} p_{k} t_{j_{k}} \cdots t_{j_{k-1}}^{-1} p_{k-1} t_{j_{k-1}} t_{j_{k}}^{-1} e=q_{r} t_{l_{r}} t_{l_{r+1}}^{-1} q_{r+1} t_{l_{r+1}} \cdots t_{l_{r-1}}^{-1} q_{r-1} t_{l_{r-1}} t_{l_{r}}^{-1} .
$$

So $t_{i_{d}}^{-1} e^{-1} p_{k} t_{j_{k}} \cdots t_{j_{k-1}}^{-1} p_{k-1} t_{j_{k-1}} t_{j_{k}}^{-1} e t_{i_{d}}=t_{i_{d}}^{-1} q_{r} t_{l_{r}} \cdots t_{h_{-1}}^{-1} q_{r-1} t_{r_{-1}} t_{l_{r}}^{-1} t_{i_{d}}$. But $t_{i_{d}}=t_{j_{k}}$ $=t_{r_{r}}$ and $e \in A_{i_{d}}, p_{k} \in A_{i_{d}}$, so this is exactly $\varphi\left(e^{-1} p_{k} \cdots p_{k-1} e\right)=\varphi\left(q_{r} \cdots q_{r-1}\right)$, and we are done as in the case with $\varepsilon=1$.

Case 2. Suppose neither $\varphi\left(h_{1}\right)$ nor $\varphi\left(h_{2}\right)$ involves a $t$-symbol. Then both $h_{1} \in A_{i_{0}}$ and $h_{2} \in A_{i_{0}}$, and $h_{1} \sim_{G} h_{2}$. By Collins' Lemma either $h_{1} \sim_{P} h_{2}$ and therefore $h_{1} \sim_{H} h_{2}$, or there exists a sequence $u_{1}, \ldots, u_{s}$ where $u_{1}=h_{1}, u_{s}=h_{2}$ in $P$ and either

(a) $u_{i+1}=x^{-1} u_{i} x$ where $x$ is $t$-free and $u_{i+1} \in B_{j}$ for some $j$, or

(b) $u_{i+1}=t_{j}^{-\varepsilon} u_{i} t_{j}^{\varepsilon}$ where $\varepsilon= \pm 1$, and this a relation of $G^{*}$. We now show $u_{i} \sim_{H} u_{i+1}$ for $i=1, \ldots, s$.

(a) If $u_{i+1}=x^{-1} u_{i} x$ then $u_{i+1} \sim_{P} u_{i}$.

(b) If $\varepsilon=1$ then $t_{j}^{-1} u_{i} t_{j}=u_{i+1}$ in $G^{*}$ and $u_{i} \in B_{j}$ for some $j \in J$ with $u_{i+1}=$ $\psi_{i}\left(u_{i}\right)$. Then $u_{i+1}=u_{i}$ in $H$.

If $\varepsilon=-1$ then $t_{j} u_{i} t_{j}^{-1}=u_{i+1}$ in $G^{*}$ and $u_{i} \in B_{i_{0}}$ with $u_{i}=\psi_{j}\left(u_{i+1}\right)$. Then $u_{i+1}=u_{i}$ in $H$. This completes the proof.

Using this lemma, we deduce Lipschutz' Theorem from [8].

TheOREM (LIPSCHUTZ). Let $H$ be the free product of groups with the solvable conjugacy problem amalgamating a cyclic subgroup generated by semicritical elements in the factors. Then $H$ has solvable conjugacy problem.

Proof. We first consider the case $H=\left\langle A_{1} * A_{2} ; a_{1}=a_{2}\right\rangle$ where $a_{i}$ is semicritical in $A_{i}, i=1,2$. Let $G^{*}=\left\langle A_{1} * A_{2}, t ; t^{-1} a_{1} t=a_{2}\right\rangle$. Since the embedding $\varphi$ : $H \rightarrow G^{*}$ defined by $\varphi\left(g_{2}\right)=g_{2}$ if $g_{2} \in A_{2}, \varphi\left(g_{1}\right)=t^{-1} g_{1} t$ if $g_{1} \in A_{1}$ is Frattini, we need only to solve the conjugacy problem in $G^{*}$. To do this, it suffices to show that $a_{1}$ and $a_{2}$ are mutually semicritical in $A_{1} * A_{2}$.

(a) This is clear.

(b) If we cyclically reduce in the free product sense, this may be reduced to the conjugacy-power-solvability in the factors.

(c) Suppose we are given $u$ and $v$ in $A_{1} * A_{2}$, and we seek a pair $(r, s)$ so that $a_{1}^{r} u a_{2}^{s}=v$. We write $u=g_{1} h_{1} \cdots g_{p} h_{p}$ in normal form (free product) with perhaps $g_{1}=1$ or $h_{p}=1$, and similarly $v=c_{1} d_{1} \cdots c_{q} d_{q}$, with $g_{i}, c_{j} \in A_{1}, h_{i}, d_{j} \in A_{2}$. We 
consider the equation

$$
d_{q}^{-1} c_{q}^{-1} \cdots d_{1}^{-1} c_{1}^{-1} a_{1}^{r} g_{1} h_{1} \cdots g_{p} h_{p} a_{2}^{s}=1 \text { in } A_{1} * A_{2} .
$$

We may determine by (a) in $A_{1}$ if there is an $r$ with $a_{1}^{r} \sim_{A} c_{1} g_{1}^{-1}$. If not, then there can be no pair $(r, s)$ with $a_{1}^{r} u a_{2}^{s}=v$. If so, $r$ is unique by (a), so, using the word problem in $A_{1}$ we determine if $a_{1}^{r}=c_{1} g_{1}^{-1}$. If this is true, we then determine if $d_{1}^{-1} h_{1}$ is trivial in $A_{2}$, and continue. Finally, if we reach the word $d_{q}^{-1} h_{p} a_{2}^{s}$, we handle this exactly as $c_{1}^{-1} a_{1}^{r} g_{1}$ was handled in the first step.

The other three cases to be considered in proving (c) are similar.

We now consider $H=\left\langle A_{0} * \ldots * A_{n} ; a_{i}=a_{0}, i=1, \ldots, n\right\rangle$ where $a_{i}$ is semicritical in $A_{i}$ for $i=0, \ldots, n$. Then the $a_{i}$ are pairwise mutually semicritical in $P=A_{0} * \cdots * A_{n}$. Let $G^{*}$ be the group $G^{*}=\left\langle P, t_{1}, \ldots, t_{n} ; t_{i}^{-1} a_{i} t_{i}=a_{0}, i=\right.$ $1, \ldots, n\rangle$. Then by the lemma the mapping $\varphi: H \rightarrow G^{*}$ with $\varphi\left(g_{0}\right)=g_{0}$ for $g_{0} \in A_{0}, \varphi\left(g_{i}\right)=t_{i}^{-1} g_{i} t_{i}$ for $g_{i} \in A_{i}, i=1, \ldots, n$, is a Frattini embedding. Thus, to solve the conjugacy problem for $H$, we need only solve the conjugacy problem for $G^{*}$. To do this, it suffices to show that $G^{*}$ may be constructed as an HNN extension of the type considered in the first part of the theorem.

Let $G^{0}=P, G^{m}=\left\langle G^{m-1}, t_{m} ; t_{m}^{-1} a_{m} t_{m}=a_{0}\right\rangle$ for $m=1, \ldots, n$. Then clearly $G^{n}=G^{*}$. To allow an iterated application of the theorem proved above for one stable letter, it remains to show that if $j>m+1$ and $a_{0}, a_{j}$ are mutually semicritical in $G^{m}$ then they are mutually semicritical in $G^{m+1}, m=0, \ldots, n-1$.

So suppose $j>m+1$ and $a_{0}, a_{j}$ are mutually semicritical in $G^{m}$. We check the three conditions in $G^{m+1}$.

(a) We know that $a_{0}$ and $a_{j}$ are not self-power-conjugate or power-conjugate in $G^{m}$. If $a_{0}^{\alpha} \sim_{G^{m+1}} a_{0}^{\beta}$ then there is a sequence $a_{0}^{\alpha}=u_{0}, u_{1}, \ldots, u_{l-1}, u_{l}=a_{0}^{\beta}$ where $u_{1}, \ldots, u_{l-1}$ in $\left\langle a_{0}\right\rangle$ and $u_{i+1}$ is obtained from $u_{i}$ by conjugating by an element of $G^{m}$ into $\left\langle a_{0}\right\rangle$ and then by $t_{m+1}^{ \pm 1}$ for $i=0, \ldots, l-1$. These conjugations cannot change the exponent of $a_{0}$. For $a_{j}$ the sequence must be completely trivial since $j>m+1$, so $a_{j}^{\alpha} \sim_{G^{m+1}} a_{j}^{\beta}$ must imply $a_{j}^{\alpha} \sim_{G^{m}} a_{j}^{\beta}$. If $a_{0}^{\alpha} \sim_{G^{m+1}} a_{j}^{\beta}$ then $a_{j}^{\beta}$ must enter into such a sequence starting with $a_{0}^{\alpha}$, which is also impossible.

(b) We must be able to determine if there exists an $\alpha$ so that $w \sim_{G^{m+1}} a_{j}^{\alpha}$ and $w \nsim_{G^{m}} a_{j}^{\alpha}$ for any $w$, and similarly for $a_{0}$. For $a_{j}$ this is similar to the argument in (a); since $j>m+1$ we cannot have $a_{j}^{\alpha}$ in a Collins' Lemma type sequence. If $w \sim_{G^{m+1}} a_{0}^{\alpha}$ but $w \nsim_{G^{m}} a_{0}^{\alpha}$, we must have $w \sim_{G^{m}} a_{m+1}^{\alpha}$, which may be decided (see Case 1 of Theorem 1).

(c) Given $u$ and $v$ in $G^{m+1}$ we must be able to decide if, for example, there are integers $r$ and $s$ with $a_{0}^{r} u a_{j}^{s}=v$ in $G^{m+1}$. We may imitate the argument for (c) above in this theorem, using Britton's Lemma in the place of the normal form theorem for free products with amalgamation.

\section{Proof of Theorem 2.}

Proof. We will assume that $h$ and $k$ have infinite order in $A$ and $B$, respectively. If either $h$ or $k$ has finite order, the argument is similar. We will say that a word $w$ of $P=A * B$ is $C$-reduced if it is reduced in $P$ in the free product sense and has no 
proper subword of the form $h k$ or $k h$. A word $w$ is cyclically $C$-reduced if every cyclic conjugate in the free product sense of $w$ is $C$-reduced.

Suppose $u \sim v$ in $C$ but $u \nsim v$ in $P$. As shown in [6], there are two possible cases, assuming that $u$ and $v$ are cyclically $C$-reduced:

Case I. $u$ and $v$ have cyclic conjugates $u^{*}$ and $v^{*}$ such that $u^{*}=h^{p} k^{q}$ and $v^{*}=h^{p^{\prime}} k^{q^{\prime}}$ for some integers $p, q, p^{\prime}, q^{\prime}$. Examining the labels of the only possible annular conjugacy diagram for $u$ and $v$ shows that the following equation holds in $C$ for some integers $p_{1}, q_{1}, \ldots, p_{n}, q_{n}$ :

$$
h^{p_{1}} k^{q_{1}} h^{p_{2}} k^{q_{2}} \cdots h^{p_{n}} k^{q_{n}} h^{-p_{n-1} k^{-q_{n-2}} \cdots h^{-p_{3}} k^{-q_{2}}=1}
$$

where $p_{1}+p_{2}=p, q_{1}=q,-p_{n}=p^{\prime}$, and $-\left(q_{n}+q_{n-1}\right)=q^{\prime}$. But then we have $p_{1}+\cdots+p_{n}-p_{n-1}-\cdots-p_{3}=0$ and $q_{1}+\cdots+q_{n}-q_{n-2}-\cdots-q_{2}=$ 0 , so $p_{1}+p_{2}=-p_{n}$ and $q_{1}=-\left(q_{n-1}+q_{n}\right)$. Thus, $u^{*}=h^{p} k^{q}$ and $v^{*}=h^{p^{\prime}} k^{q^{\prime}}$ are conjugate if and only if $p=p^{\prime}$ and $q=q^{\prime}$.

Case II. $u$ and $v$ have cyclic conjugates $u^{*}$ and $v^{*}$ having one of the following forms:

(i) $u^{*}=u_{1} k^{q_{1}} \cdots u_{n} k^{q_{n}}, v^{*}=v_{1} k^{q_{1}} \cdots v_{n} k^{q_{n}}$ where $u_{i}, v_{i} \in A-\langle h\rangle$ and $q_{1}, \ldots, q_{n}$ are nonzero integers, or

(ii) $u^{*}=u_{1} h^{p_{1}} \cdots u_{n} h^{p_{n}}, v^{*}=v_{1} h^{p_{1}} \cdots v_{n} h^{p_{n}}$ where $u_{i}, v_{i} \in B-\langle k\rangle$ and $p_{1}, \ldots, p_{n}$ are nonzero integers.

We will consider words of form (i). Then $u \sim v$ if and only if the following system has a integral solution $\left(x_{1}, \ldots, x_{n}\right)$ :

$$
\begin{array}{ccc}
h^{x_{1}} u_{2} \cdots u_{n} h^{-x_{n}} & = & v_{2} \cdots v_{n} \\
h^{x_{2}} u_{3} \cdots u_{1} h^{-x_{1}} & = & v_{3} \cdots v_{1} \\
\vdots & & \vdots \\
h^{x_{n-1}} u_{n} \cdots u_{n-2} h^{-x_{n-2}} & = & v_{n} \cdots v_{n-2} \\
h^{x_{n}} u_{1} \cdots u_{n-1} h^{-x_{n-1}} & = & v_{1} \cdots v_{n-1}
\end{array}
$$

We abbreviate $u_{i} \cdots u_{i-2}$ and $v_{i} \cdots v_{i-2}$ (modulo $n$, of course) by $U_{i}$ and $V_{i}$, respectively. Thus, we have the system $h^{x_{i+1}} U_{i} h^{-x_{i}}=V_{i}$ for $i=1, \ldots, n$, where $x_{n+1}=x_{1}$. But the algorithm given in Theorem 1 to determine if system (**) has a solution $\left(\alpha_{1}, \ldots, \alpha_{n}\right)$ can be used to determine if this system has a solution $\left(x_{1}, \ldots, x_{n}\right)$.

\section{REFERENCES}

1. M. Anshel, Conjugate powers in HNN groups, Proc. Amer. Math. Soc. 54 (1976), 19-23. MR 52 \#14059.

2. M. Anshel and P. Stebe, The solvability of the conjugacy problem for certain HNN groups, Bull. Amer. Math. Soc. 80 (1974), 266-270.

3. D. J. Collins, On recursively enumerable degrees and the conjugacy problem, Acta Math. 122 (1969), 115-160. MR 39 \#4001.

4. L. P. Comerford and B. Truffault, The conjugacy problem for free products of sixth-groups with cyclic amalgamation, Math. Z. 149 (1976), 169-181. MR 53 \# 13418. 
5. R. D. Hurwitz, On the conjugacy problem in certain product groups, $\mathrm{Ph}$. D. Thesis, University of Illinois, Urbana, 1974.

6. On the conjugacy problem in a free product with commuting subgroups, Math. Ann. 221 (1976), 1-8. MR 54 \#414.

7. S. Lipschutz, Generalization of Dehn's result on the conjugacy problem, Proc. Amer. Math. Soc. 17 (1966), 759-762. MR 33 \#5706.

8. The conjugacy problem and cyclic amalgamations, Bull. Amer. Math. Soc. 81 (1975), 114-116. MR 52 \#580.

Department of Mathematics, Skidmore College, Saratoga Springs, New York 12866 